\title{
Transformationen des Historischen
}

\author{
Geschichtserfahrung und Geschichtsschreibung bei Ferdinand Gregorovius \\ Hrsg. v. Dominik Fugger
}

Transformationen des Historischen Herausgegeben von Dominik Fugger

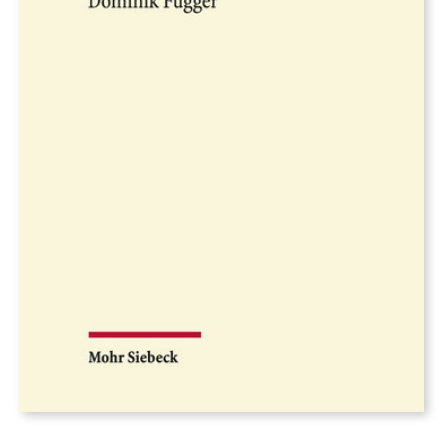

2015. VII, 140 Seiten.

ISBN 978-3-16-153835-3 DOI 10.1628/978-3-16-153835-3 eBook PDF 54,00€

ISBN 978-3-16-153834-6

Leinen $54,00 €$
Die Beiträger dieses Bandes möchten Geschichtsschreibung als erfahrungsbezogene und erfahrungsgenerierende Praxis am Beispiel von Ferdinand Gregorovius (1821-1891) verstehbar machen. Ausgangspunkt ist hierbei die individuelle Erfahrung von Geschichtlichkeit.

Gregorovius' unmittelbare, wenn auch sehr voraussetzungsreiche Anverwandlung der Geschichte bildet die erste der titelgebenden »Transformationen des Historischen«. Ohne einen tieferen Blick auf die ideengeschichtlichen Bedingungen ist ein solches Geschichtserlebnis nicht zu verstehen. Soziale Wirksamkeit kann es nur in produktiver Umsetzung entfalten, denn nur auf diese Weise lässt es sich mitteilen. Dies ist die zweite Transformation des Historischen. Der Innovationsgrad der dadurch angeregten Werke, ihre Wahrnehmung durch die Zeitgenossen und ihre lebenspraktischen Folgen für den Geschichtsschreiber zeigen die kulturelle Bedeutung in der Zeit. Die folgenden Generationen können sich zu einem solchen Zugang ihrerseits nur historisch verhalten; um ihn erneut produktiv werden zu lassen, bedarf es weiterer Transformationen. Sie sind Gegenstand der Rezeptionsgeschichte. Die hier versammelten Beiträge widmen sich diesen Aspekten mit dem Ziel, einen Blick auf eine individuelle wie gesellschaftliche Funktion von Geschichte jenseits unmittelbarer diskursiver Inanspruchnahme freizugeben.

\section{Inhaltsübersicht}

Dominik Fugger: Erlösung durch Verehrung und Arbeit. Ferdinand Gregorovius und die Geschichte als existentielle Erfahrung - Jens Halfwassen: Gregorovius als Leser Plotins - Peter Kuhlmann: Ferdinand Gregorovius' Hadrian-Geschichte im Kontext ihrer Zeit - Wolfgang Struck: Zum Raum wird hier die Zeit. Gregorovius' Corsica - Julia Ilgner: Der Biograph Ferdinand Gregorovius und seine literarische Rezeption - Karsten Lorek: Legendarische Wirklichkeit und erdichtete Geschichte. Überlegungen zur Gregorovius-Rezeption in Thomas Manns Gregorius-Roman Der Erwäh/te

Dominik Fugger Geboren 1975; Studium der Geschichte und Germanistik; 2001 MA; 2006 Promotion; seit 2008 am MaxWeber-Kolleg der Universität Erfurt; seit 2014 Vertretung einer W1-Professur »Verflechtungsgeschichte« an der Kollegforschergruppe »Religiöse Individualisierung in historischer Perspektive".

Jetzt bestellen:

https://mohrsiebeck.com/buch/transformationen-des-historischen-9783161538353?no_cache=1 order@mohrsiebeck.com

Telefon: $+49(0) 7071-923-17$

Telefax: +49 (0)7071-51104 